\title{
Identification of differentially expressed genes in clear cell renal cell carcinoma by analysis of full-length enriched cDNA library
}

\author{
Sai-Wen Tang ${ }^{1}$, Wen-Hsin Chang ${ }^{1}$, Yu-Wei Chao ${ }^{1}$, Chung-Yei Lin ${ }^{1}$, Hsiao-Fen Chen ${ }^{1}$, \\ Yen-Han Lai ${ }^{1}$, Bo-Wen Zhan ${ }^{1}$, Yih-Ching Su${ }^{1}$, Sen-Wen Jane ${ }^{1}$, Yu-Chi Chen ${ }^{1}$, \\ Chyong-Ing Hsu ${ }^{1}$, Wei-Chou Lin ${ }^{2}$, Kao-Chung Wang ${ }^{3}$, Ming-Kuen Lai ${ }^{3}$ \& \\ Jung-Yaw Lin ${ }^{1, *}$ \\ ${ }^{1}$ Institute of Biochemistry and Molecular Biology, College of Medicine, National Taiwan University, Taipei, \\ Taiwan, R.O.C; ${ }^{2}$ Department of Pathology, National Taiwan University Hospital, Taipei, Taiwan, R.O.C; \\ ${ }^{3}$ Department of Urology, National Taiwan University Hospital, Taipei, Taiwan, R.O.C
}

(C) 2006 National Science Council, Taipei

Key words: clear cell renal cell carcinoma, full-length cDNA library, Annexin A2, argininosuccinate synthetase

\begin{abstract}
Summary
Renal cell carcinoma (RCC) is the most common malignancy in adult kidney, and accounts for $3 \%$ of malignancies worldwide with increasing incidence. Clear cell RCC (ccRCC) is the major type in RCC. Resection by surgery is the main treatment because the response of ccRCC to traditional therapies is very poor. To identify the tumor-associated genes for better understanding the molecular mechanism of ccRCC, the full-length enriched cDNA libraries of ccRCC and normal kidney tissues were constructed by the oligo-capping method. Nucleotide sequences of the cDNA libraries of ccRCC and normal kidney tissues were sequenced. From the sequence analysis of 19,425 and 12,400 clones of ccRCC and normal kidney tissues, 4356 and 3055 genes were identified, respectively. By comparing the gene-expression patterns of ccRCC and normal tissues, the up- or down-regulated genes were identified. Among these identified genes, the differential expression of annexin A2 and argininosuccinate synthetase genes were further confirmed by quantitative real-time PCR and Western blot analysis.
\end{abstract}

\section{Introduction}

Renal cell carcinoma (RCC) represents $3 \%$ of all malignancies and the third most common urological cancer of men [1]. RCC affects approximately 150,000 people worldwide each year, causing close to 78,000 deaths annually [2]. One third of the RCC patients develop metastasis at presentation with a poor 5-year survival because there is no

\footnotetext{
*To whom correspondence should be addressed. Tel: + 886-223123456; Fax: +886-2-23415334; E-mail: jylin@ha.mc.ntu. edu.tw
}

early warning sign [3]. At least five types of RCC were found, and the majority $(>80 \%)$ are classified as clear cell RCC (ccRCC) [4].

Until now, there is no useful tumor marker for diagnosis and follow-up of RCC. RCC is one of the most therapy-resistant cancers and has poor response to chemotherapy, hormonal therapy and radiation therapy [5]. So, resection of kidney is the main treatment for RCC but effective only in about $70 \%$ of early-stage and localized RCC [6].

To investigate the molecular mechanism of ccRCC, the full-length cDNA libraries of ccRCC and normal kidney tissues were constructed by the 
oligo-capping method [7, 8]. By comparison of the gene expression of the ccRCC and normal kidney, we identified 201 up-regulated and 182 down-regulated genes. Among these differentially expressed genes, the degrees of up- or down-expression of annexin A2 (ANXA2) and argininosuccinate synthetase (ASS) genes were further investigated by quantitative realtime PCR and Western blot analysis.

\section{Materials and methods}

\section{Tissue collection}

Tissues of ccRCC and adjacent normal kidney were obtained from 45 patients at the Department of Urology of the National Taiwan University Hospital. After surgical resection, the samples were immediately snap frozen by liquid nitrogen and then stored at $-80{ }^{\circ} \mathrm{C}$.

\section{Purification of total RNA and $m R N A$}

Total RNAs were isolated from ccRCC and normal kidney tissues using Trizol reagent (Invitrogen) and RNeasy (Qiagen), and the mRNAs were purified by oligo-dT cellulose (MRC) according to the manufacturer's instructions. The integrity of the mRNAs was confirmed by agarose gel electrophoresis.

\section{Construction of full-length cDNA library}

The oligo-capping method was performed as described previously [29]. Briefly, the phosphate of the 5'-ends of truncated mRNAs was hydrolyzed by bacterial alkaline phosphatase (Takara). After treatment of tobacco acid pyrophosphatase (Wako), the cap structure of the intact mRNAs was removed and resulted in a $5^{\prime}$-end phosphate group. Then a $5^{\prime}$-oligo adaptor primer was ligated to the $5^{\prime}$-end phosphate group by T4 RNA ligase (Takara). The oligo-capped mRNAs were reversetranscribed by Superscript ${ }^{\mathrm{TM}}$ II Reverse Transcriptase (Invitrogen) to synthesize the first-strand cDNAs with the oligo-dT adapter primer. The first-strand cDNA was amplified by PCR and digested with SfiI restriction enzyme (New England Biolabs). After cloning into DraIII-digested pME18S-FL3 vector, the full-length enriched cDNA library was transformed into $E$. coli strain TOP10 by electroporation (Bio-Rad Gene Pluser).
The colonies were sequenced by ABI 3700 sequencer and blasted with NCBI database (http://www.ncbi.nlm.nih.gov/BLAST/).

\section{Quantitative real-time PCR}

Total RNAs were isolated by using Trizol reagent and treated with RNase-free DNase I (Takara). Single-stranded cDNAs were synthesized by SuperScript $^{\mathrm{TM}}$ II Reverse Transcriptase (Invitrogen). The primer pairs for ANXA2 and ASS genes were designed by using Beacon Designer 3 (Table 1). Quantitative real-time PCR was performed using iCycler (Bio-Rad), and threshold cycle number was determined using iCycler software version 2.3. The PCR condition comprised an initial denature step at $95{ }^{\circ} \mathrm{C}$ for $15 \mathrm{~min}$ followed by 50 cycles at $95{ }^{\circ} \mathrm{C}$ for $10 \mathrm{~s}$ and $60{ }^{\circ} \mathrm{C}$ for $45 \mathrm{~s}$. Amplification specificity was confirmed by generating melting curve following the manufacturer's instructions. Reactions were performed in duplicates for each data point, and threshold cycle numbers were averaged. The results were normalized to tumor protein translationally-controlled 1 (TPT1) which had minimal variation in all ccRCC and normal kidney tissues in this experiment. The relative expression level was calculated by $2^{(-\Delta \Delta \mathrm{Ct})}$ Method [9].

\section{Western blotting}

Anti-ANXA2 and anti-ASS monoclonal antibodies were obtained from BD Biosciences Pharmigen and anti-actin monoclonal antibody was from Chemicon. The proteins of RCC and normal kidney tissues lysates were electrophoresed through a $12.5 \%$ SDS-PAGE, electroblotted to PVDF membrane and then detected by using appropriate primary antibody, horseradish-perox-

Table 1. Primers for quantitative real-time PCR analysis.

\begin{tabular}{ll}
\hline $\begin{array}{l}\text { TPT1 forward } \\
\text { primer }\end{array}$ & GATCGCGGACGGGTTGT \\
TPT1 reverse & TTCAGCGGAGGCATTTCC \\
primer & \\
ANXA2 forward & GATGCTCGGGATCTCTATGAC \\
primer & \\
ANXA2 reverse & TAAGGGCTGTAACTCTTGTACC \\
primer & \\
ASS forward primer & AGACATCGAGGCCTTCACCA \\
ASS reverse primer & GGCTAGGCCGTAAACCGGTA
\end{tabular}


idase secondary antibody and Enhanced Chemiluminescence (ECL) reagent (Amersham Bioscience). The quantification of protein expression was performed with AlphaImagerTM 2200 documentation Analysis system (Alphainnotech).

\section{Results}

\section{Analysis of gene expression profiles of ccRCC and} normal kidney

To obtain the gene expression profiles in ccRCC and normal kidney, we constructed oligo-capping cDNA libraries from primary ccRCC and normal kidney tissues. After transforming into TOP10, 19,425 and 12,400 clones were picked and sequenced from RCC and normal kidney cDNA libraries, respectively. By using the BLAST program with the UniGene database of NCBI, we identified 4356 and 3055 genes for the cDNA libraries from primary ccRCC and normal kidney tissues, respectively. The gene expression pattern in the libraries of ccRCC and normal kidney was compared to identify up- or down-regulated genes. We defined genes which clone number in the library of ccRCC was three-fold higher than that in the library of normal kidney as up-regulated genes, and genes which clone number in the library
Table 2. Functional classification of the up- and downregulated genes.

\begin{tabular}{lll}
\hline Function & $\begin{array}{l}\text { Number of } \\
\text { up-regulated } \\
\text { genes }\end{array}$ & $\begin{array}{l}\text { Number of } \\
\text { down-regulated } \\
\text { genes }\end{array}$ \\
\hline Apoptosis & 3 & 3 \\
Cell growth & 9 & 7 \\
Cytoskeleton & 9 & 19 \\
DNA binding & 3 & 5 \\
Extracellular matrix protein & 1 & 2 \\
Growth factor & 1 & 2 \\
Immune & 16 & 4 \\
Membrane protein & 6 & 4 \\
Metabolism & 51 & 23 \\
Protease inhibitor & 2 & 3 \\
Protein degradation & 7 & 5 \\
RNA processing & 7 & 11 \\
Signal transduction & 33 & 22 \\
Transcription & 5 & 10 \\
Translation & 13 & 25 \\
Transport & 15 & 15 \\
Chaperon & 5 & 0 \\
Angiogenesis & 1 & 0 \\
Mitochodria & 0 & 3 \\
Development & 0 & 2 \\
Unknown & 14 & 17 \\
Total & 201 & 182 \\
\hline
\end{tabular}

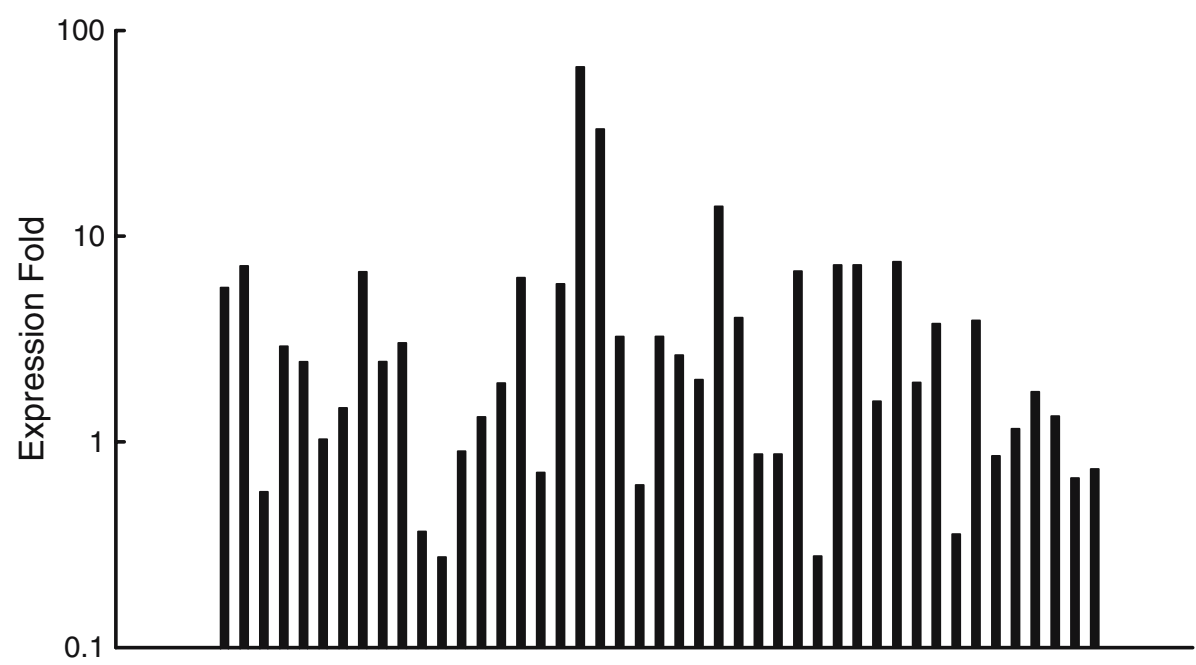

Patients

Figure 1. Up-regulation of ANXA2 mRNA in forty-five pairs of ccRCC tissues. Quantitative Real-time PCR analysis was performed for the expression of ANXA2 in ccRCC tissue pairs, and each bar represents the ANXA2 expression fold of one ccRCC tumor. Nineteen ccRCC tumors (42\%) had at least 3-fold up-regulation of ANXA2 mRNA as compared with that of adjacent normal tissues. 

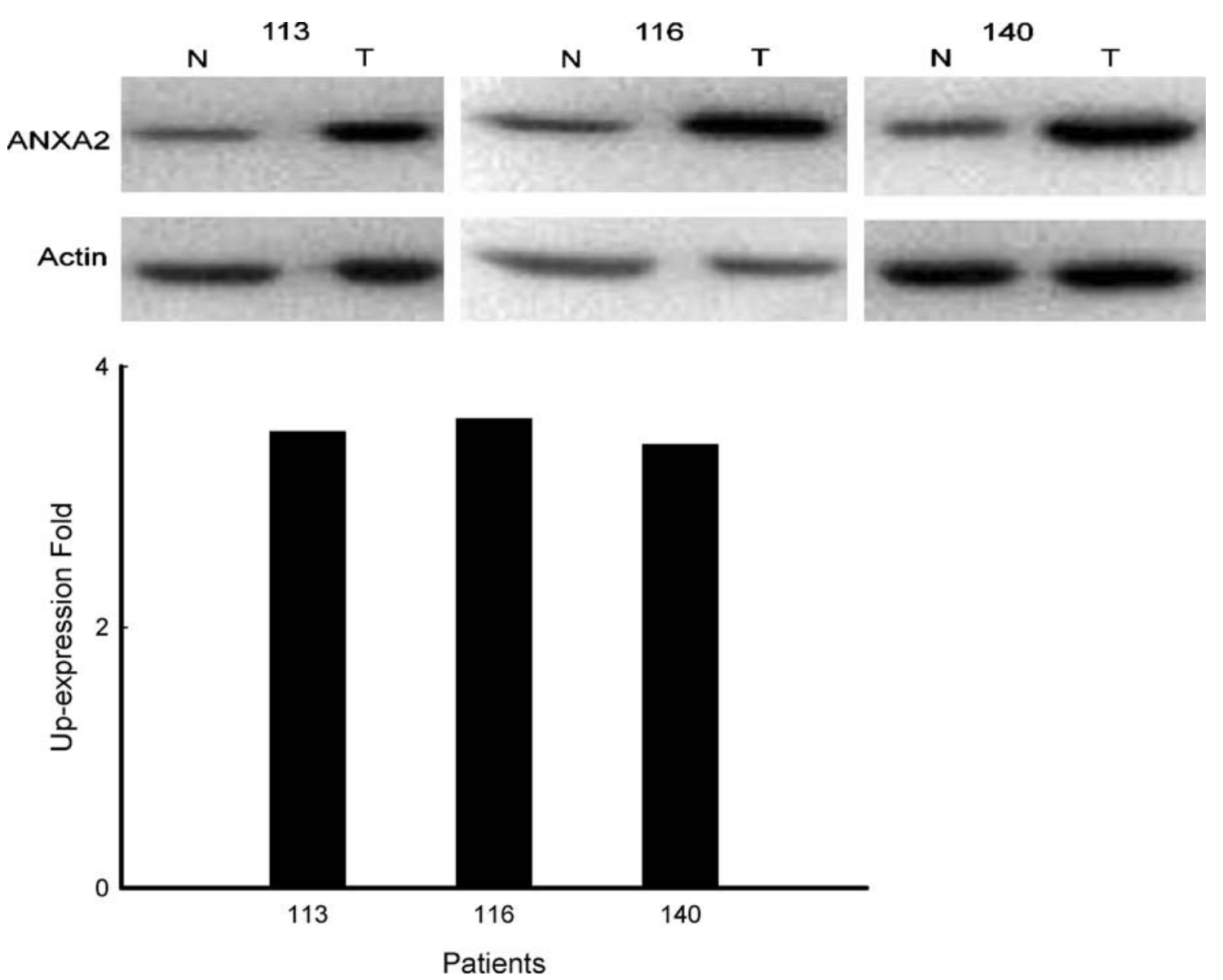

Figure 2. Up-regulation of ANXA2 protein in ccRCC tissue pairs. The up-regulation of ANXA2 protein was shown in ccRCC sample 113, 116 and 140. Whole tissue lysates from ccRCC tissues and adjacent normal tissues were separated by SDS-PAGE and analyzed for ANXA2 protein expression with anti-ANXA2 monoclonal antibody by Western blotting. Actin is shown as a loading control. The up-regulation fold of ANXA2 protein in ccRCC tissues was shown by comparing with ANXA2 protein level of normal tissues.

of normal kidney was three-fold lower than that in the library of ccRCC as down-regulated genes, and 201 up-regulated and 182 down-regulated genes were identified.

\section{Functional classification of the differentially expressed genes}

These up- or down-regulated genes were classified into groups by functions (Table 2). We observed obvious changes in the gene expressions of cytoskeleton, immune, metabolism, signal transduction, translation, and chaperon related genes between ccRCC and normal kidney tissues. However, the significance of the alterations in these genes needed to be investigated. In addition, many identified genes which have been reported as cancer-associated genes. For example, the up-expression of cyclin D3 gene in liver metastasis of colorectal cancer [10], matrix metallopeptidase 7 in oral squamous cell carcinoma [11], and regulator of G-protein signaling 5 in RCC [12] were observed and they were also identified as up-regulated genes in this study. Furthermore, the association of the apoptosis-related gene kininogen 1 [13] and the cytoskeleton-related gene gelsolin [14] with cancer were previously reported and the down-regulation of kininogen 1 and gelsolin in ccRCC tissues was also found in this study. These observations indicated that the identified genes by the full-length cDNA library method might be related with ccRCC progression. 


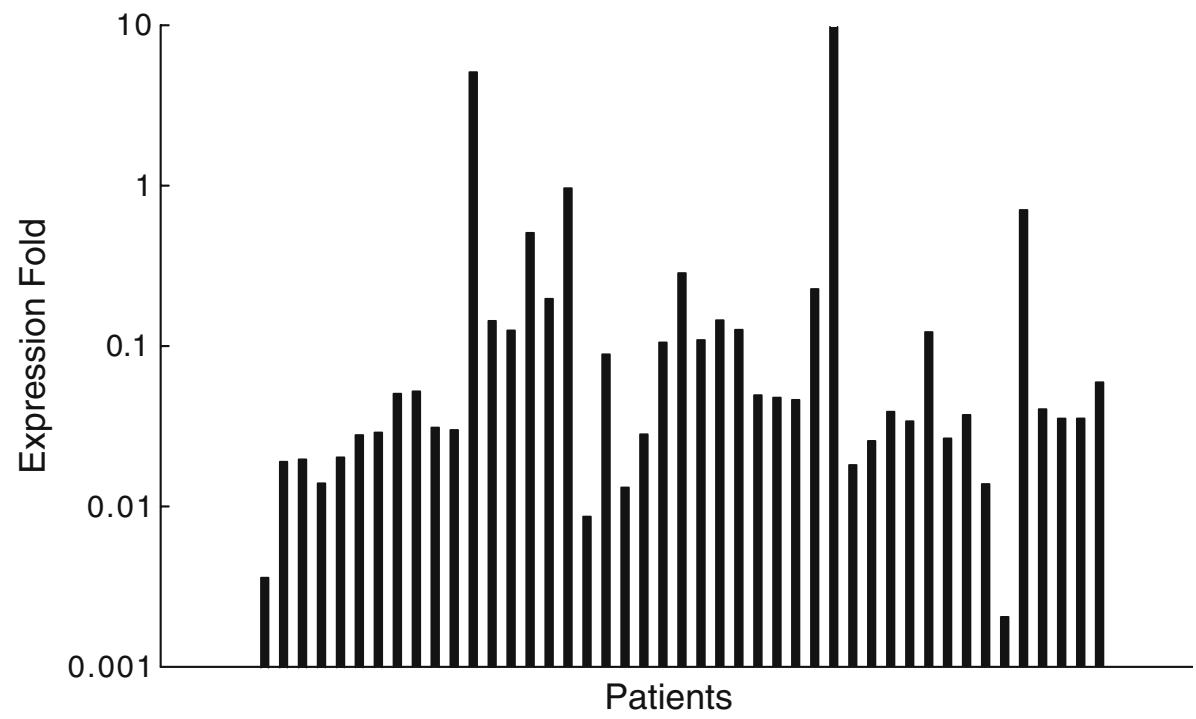

Figure 3. Down-regulation of ASS mRNA in forty-five pairs of ccRCC tissues. ASS gene expression was analyzed by quantitative Real-time PCR analysis in ccRCC tissue pairs, and each bar represents the ASS expression fold of one ccRCC tumor. Forty-one ccRCC tumors $(91 \%)$ had at least 3-fold down-regulation of ASS as compared with that of normal tissues.

\section{Differential expression of ANXA2 and ASS genes in ccRCC tissue pairs}

The up-regulated gene ANXA2 and the downregulated gene ASS were subjected to further study. Quantitative real-time PCR analysis of ccRCC tissue pairs was carried out, and the results showed that the expression levels of ANXA2 gene had an average of 4.5 -fold up-regulation in ccRCC tissues (Figure 1); then by Western blot analysis, we found that ANXA2 protein level was up-regulated 3.5, 3.6 and 3.4-folds in ccRCC tissue pair samples 113, 116 and 140 respectively (Figure 2). The down-expression of ASS mRNA of ccRCC tissues was also demonstrated in most tissue pairs, and the average down-regulation is 70.2-fold (Figure 3). The down-regulation of ASS protein was also observed in ccRCC tissue pairs 103, 137 and 150, and the degree of down-regulation is $27.7,10.3$ and 6.4 -fold, respectively (Figure 4).

\section{Discussion}

Until now, this is the first report to analyze the gene expression profiles of ccRCC and normal kidney by construction of full-length cDNA library. This method provides more precise measurement of gene expression than the technology of cDNA microarray, which is usually performed to identify differentially expressed genes $[15,16]$, and more convenient acquirement of the full-length cDNAs for further studying than the analysis of cDNA subtraction $[17,18]$. In this study, we identified a group of up- or down-regulated genes by analysis of full-length cDNA libraries, and found significant variations between the gene expression profiles of ccRCC and normal kidney tissues.

The up-regulation of ANXA2 and the downregulation of ASS were demonstrated by real-time PCR and Western blot analysis in ccRCC tissues. Therefore, by the analysis of full-length cDNA libraries we were able to identify the differentiallyexpressed genes in cancer tissues. However, more identified genes should be further confirmed. Annexin A2 was found in transformed cells as a substrate of $p p 60^{\text {src }}$ tyrosine kinase [19], and annexin A2 up-expression is observed in pancreatic adenocarcinoma [20], human hepatocellular carcinoma [21], and a multidrug-resistant, smallcell lung cancer cell line [22]. Annexin A2 is part of the primer recognition protein complex that interacts with DNA polymerase $\alpha$ in lagging strand DNA replication [23]. In addition, antisense oligonucleotides to annexin A2 retard proliferation of Hela cells [24], and the expression of annexin A2 

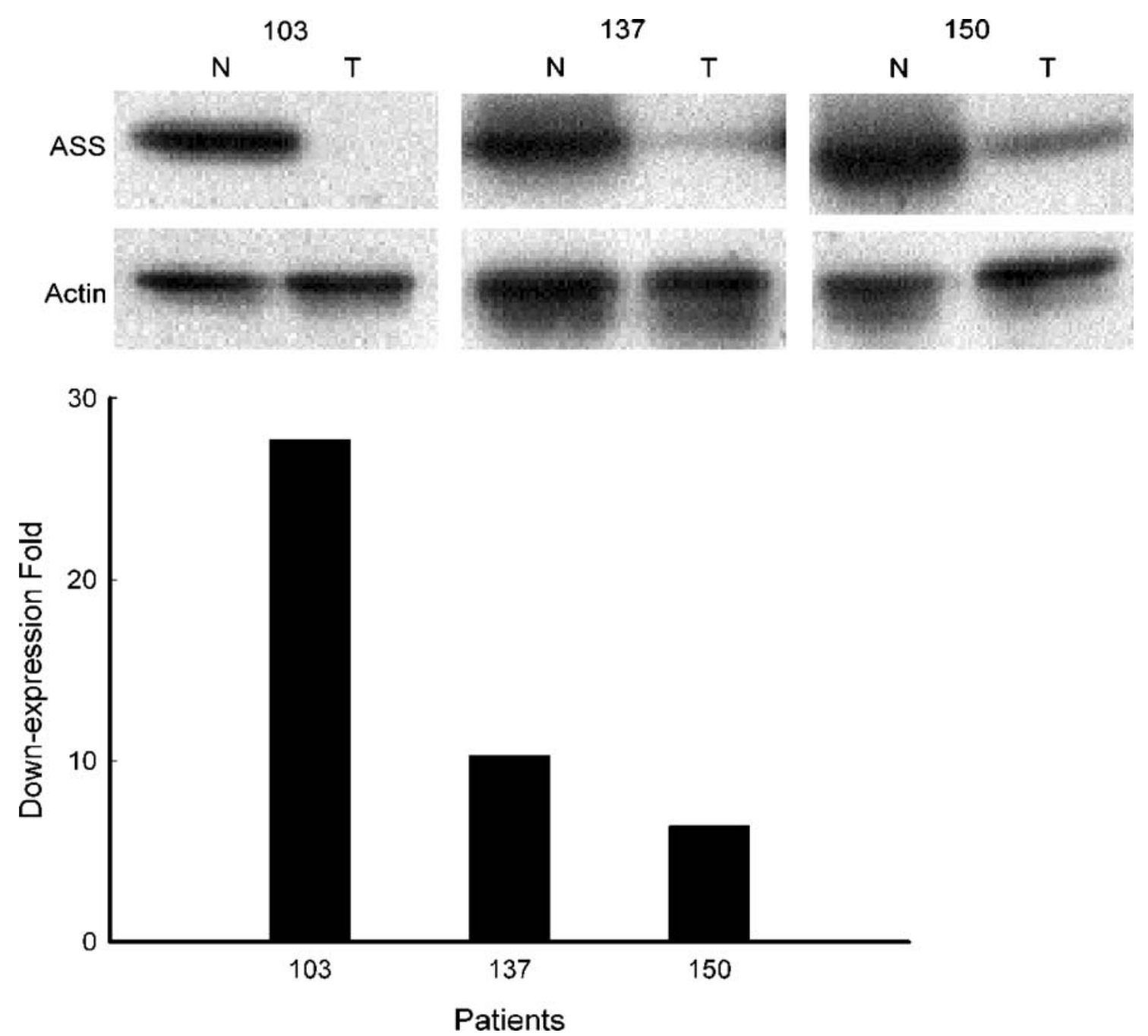

Figure 4. Down-regulation of ASS protein in ccRCC tissue pairs. The down-regulation of ASS protein was shown in ccRCC sample 103, 137 and 150. Whole tissue lysates from ccRCC tissues and adjacent normal tissues were separated by SDS-PAGE and analyzed for ASS protein expression with anti-ASS monoclonal antibody by Western blotting. Actin is shown as a loading control. The down-regulation fold of ASS protein in ccRCC tissues was shown by comparing with ASS protein level of normal tissues.

is regulated during the mammalian cell cycle [25]. Recent study has demonstrated ANXA2 is a RNA-binding protein that interacts with the mRNA of the nuclear oncogene, c-myc, and results in an increase in c-Myc protein [26]. Therefore, the up-regulation of ANXA2 may contribute to tumorigenesis of ccRCC through increasing the c-Myc protein level.

ASS is the first of two enzymes to convert citrulline to arginine. This pathway allowed cells to synthesize arginine from citrulline, making this amino acid nonessential for the growth of most mammalian cells. Many tumors, such as melanoma, hepatocellular carcinoma, and prostate carcinoma, showed significant down-regulation of ASS [27]. Surprisingly, it has been reported that ASS was associated with the oncogene, A-Raf. However, the role of ASS in carcinogenesis of ccRCC is not clear and should be studied.

It has been reported that metabolism genes, for example proline oxidase [28, 29] and cytosolic phospholipase A2 [30, 31], were differentially expressed in tumor cells and might have a role in carcinogenesis. Many metabolism genes with abnormal expression in ccRCC tissues, including ASS, were identified by our investigation. The 
phenomenon might be explained by that cancer cells have loss their normal functions or some metabolism genes have another role in cancer cells. Thus, it is worth to be studied how these genes are associated with tumor progression.

The up-regulation of chaperones has been reported as a common feature of human cancers [32], including ovarian cancer [33], breast cancer [34], oral cancer [35], and renal cancer [36]. Similarly, we also identified five genes with chaperone function as up-regulated genes in this study. The correlation between these genes and carcinogenesis of ccRCC needs to be further investigated.

High percentage of ccRCC patients develops metastasis finally even though the patients had no sign of metastasis at first presentation [37]. Cell migration is a critical step for metastasis, and thus control of cell migration via the actin cytoskeleton provides the possibility for regulation of metastasis [38]. We identified 9 up-regulated and 19 downregulated genes with cytoskeleton-related functions. Among the cytoskeleton-related genes, several genes, such as gelsolin gene [39], play a role in regulation of actin polymerization and might be potential targets for ccRCC metastasis.

\section{References}

1. Pantuck A.J., Zisman A. and Belldegrun A.S., The changing natural history of renal cell carcinoma. J. Urol. 166(5): 1611-1623, 2001.

2. Pavlovich C.P. and Schmidt L.S., Searching for the hereditary causes of renal-cell carcinoma. Nat. Rev. Cancer 4(5): 381-393, 2004

3. Atkins D., Lichtenfels R. and Seliger B., Heat shock proteins in renal cell carcinomas. Contrib. Nephrol. 148: 35-56, 2005.

4. Cheville J.C., Lohse C.M., Zincke H., Weaver A.L. and Blute M.L., Comparisons of outcome and prognostic features among histologic subtypes of renal cell carcinoma. Am. J. Surg. Pathol. 27(5): 612-624, 2003.

5. Amato R.J., Chemotherapy for renal cell carcinoma. Semin. Oncol. 27(2): 177-186, 2000.

6. Russo P., Renal cell carcinoma: presentation, staging, and surgical treatment. Semin. Oncol. 27(2): 160-176, 2000.

7. Suzuki Y. and Sugano S., Construction of a full-length enriched and a $5^{\prime}$-end enriched cDNA library using the oligo-capping method. Methods Mol. Biol. 221: 73-91, 2003.

8. Suzuki Y., Yoshitomo-Nakagawa K., Maruyama K., Suyama A. and Sugano S., Construction and characterization of a full length-enriched and a $5^{\prime}$-end-enriched cDNA library. Gene 200(1-2): 149-156, 1997.

9. Livak K.J. and Schmittgen T.D., Analysis of relative gene expression data using real-time quantitative PCR and the
2(-Delta Delta C(T)) Method. Methods 25(4): 402-408, 2001.

10. Tanami H., Tsuda H., Okabe S., Iwai T., Sugihara K., Imoto I. and Inazawa J., Involvement of cyclin D3 in liver metastasis of colorectal cancer, revealed by genome-wide copy-number analysis. Lab. Invest. 85(9): 1118-1129, 2005.

11. Snijders A.M., Schmidt B.L., Fridlyand J., Dekker N., Pinkel D., Jordan R.C. and Albertson D.G., Rare amplicons implicate frequent deregulation of cell fate specification pathways in oral squamous cell carcinoma. Oncogene 24(26): 4232-4242, 2005.

12. Furuya M., Nishiyama M., Kimura S., Suyama T., Naya Y., Ito H., Nikaido T. and Ishikura H., Expression of regulator of $\mathrm{G}$ protein signalling protein 5 (RGS5) in the tumour vasculature of human renal cell carcinoma. J. Pathol. 203(1): 551-558, 2004.

13. Kawasaki M., Maeda T., Hanasawa K., Ohkubo I. and Tani T., Effect of His-Gly-Lys motif derived from domain 5 of high molecular weight kininogen on suppression of cancer metastasis both in vitro and in vivo. J. Biol. Chem. 278(49): 49301-49307, 2003.

14. Visapaa H., Bui M., Huang Y., Seligson D., Tsai H., Pantuck A., Figlin R., Rao J.Y., Belldegrun A., Horvath S. and Palotie A., Correlation of Ki-67 and gelsolin expression to clinical outcome in renal clear cell carcinoma. Urology 61(4): 845-850, 2003.

15. Amatschek S., Koenig U., Auer H., Steinlein P., Pacher M., Gruenfelder A., Dekan G., Vogl S., Kubista E., Heider K.H., Stratowa C., Schreiber M. and Sommergruber W., Tissuewide expression profiling using cDNA subtraction and microarrays to identify tumor-specific genes. Cancer Res. 64(3): 844-856, 2004

16. Planaguma J., Diaz-Fuertes M., Gil-Moreno A., Abal M., Monge M., Garcia A., Baro T., Thomson T.M., Xercavins J., Alameda F. and Reventos J., A differential gene expression profile reveals overexpression of RUNX1/AML1 in invasive endometrioid carcinoma. Cancer Res. 64(24): 88468853, 2004.

17. Baba I., Shirasawa S., Iwamoto R., Okumura K., Tsunoda T., Nishioka M., Fukuyama K., Yamamoto K., Mekada E. and Sasazuki T., Involvement of deregulated epiregulin expression in tumorigenesis in vivo through activated Ki-Ras signaling pathway in human colon cancer cells. Cancer Res. 60(24): 6886-6889, 2000.

18. Jiang Y., Harlocker S.L., Molesh D.A., Dillon D.C., Stolk J.A., Houghton R.L., Repasky E.A., Badaro R., Reed S.G. and Xu J., Discovery of differentially expressed genes in human breast cancer using subtracted cDNA libraries and cDNA microarrays. Oncogene 21(14): 2270 2282, 2002.

19. Klee C.B., Ca2 + -dependent phospholipid- (and membrane-) binding proteins. Biochemistry 27(18): 6645-6653, 1988.

20. Kumble K.D., Hirota M., Pour P.M. and Vishwanatha J.K., Enhanced levels of annexins in pancreatic carcinoma cells of Syrian hamsters and their intrapancreatic allografts. Cancer Res. 52(1): 163-167, 1992.

21. Frohlich M., Motte P., Galvin K., Takahashi H., Wands J. and Ozturk M., Enhanced expression of the protein kinase substrate p36 in human hepatocellular carcinoma. Mol. Cell. Biol. 10(6): 3216-3223, 1990.

22. Cole S.P., Pinkoski M.J., Bhardwaj G. and Deeley R.G., Elevated expression of annexin II (lipocortin II, p36) in a multidrug resistant small cell lung cancer cell line. Br. J. Cancer 65(4): 498-502, 1992. 
23. Jindal H.K., Chaney W.G., Anderson C.W., Davis R.G. and Vishwanatha J.K., The protein-tyrosine kinase substrate, calpactin I heavy chain (p36), is part of the primer recognition protein complex that interacts with DNA polymerase alpha. J. Biol. Chem. 266(8): 5169-5176, 1991.

24. Kumble K.D., Iversen P.L. and Vishwanatha J.K., The role of primer recognition proteins in DNA replication: inhibition of cellular proliferation by antisense oligodeoxyribonucleotides. J. Cell. Sci. 101(Pt 1): 35-41, 1992.

25. Chiang Y., Schneiderman M.H. and Vishwanatha J.K., Annexin II expression is regulated during mammalian cell cycle. Cancer Res. 53(24): 6017-6021, 1993.

26. Filipenko N.R., MacLeod T.J., Yoon C.S. and Waisman D.M., Annexin A2 is a novel RNA-binding protein. J. Biol. Chem. 279(10): 8723-8731, 2004.

27. Dillon B.J., Prieto V.G., Curley S.A., Ensor C.M., Holtsberg F.W., Bomalaski J.S. and Clark M.A., Incidence and distribution of argininosuccinate synthetase deficiency in human cancers: a method for identifying cancers sensitive to arginine deprivation. Cancer 100(4): 826-833, 2004.

28. Maxwell S.A. and Rivera A., Proline oxidase induces apoptosis in tumor cells, and its expression is frequently absent or reduced in renal carcinomas. J. Biol. Chem. 278(11): 9784-9789, 2003.

29. Rivera A. and Maxwell S.A., The p53-induced gene-6 (proline oxidase) mediates apoptosis through a calcineurindependent pathway. J. Biol. Chem. 280(32): 29346-29354, 2005.

30. Blaine S.A., Wick M., Dessev C. and Nemenoff R.A., Induction of cPLA2 in lung epithelial cells and non-small cell lung cancer is mediated by Spl and c-Jun. J. Biol. Chem 276(46): 42737-42743, 2001.
31. Dimberg J., Samuelsson A., Hugander A. and Soderkvist P., Gene expression of cyclooxygenase-2, group II and cytosolic phospholipase A2 in human colorectal cancer. Anticancer Res. 18(5A): 3283-3287, 1998.

32. Whitesell L. and Lindquist S.L., HSP90 and the chaperoning of cancer. Nat. Rev. Cancer 5(10): 761-772, 2005.

33. Kimura E., Enns R.E., Alcaraz J.E., Arboleda J., Slamon D.J. and Howell S.B., Correlation of the survival of ovarian cancer patients with mRNA expression of the $60-\mathrm{kD}$ heatshock protein HSP-60. J. Clin. Oncol. 11(5): 891-898, 1993.

34. Ciocca D.R., Clark G.M., Tandon A.K., Fuqua S.A., Welch W.J. and McGuire W.L., Heat shock protein hsp70 in patients with axillary lymph node-negative breast cancer: prognostic implications. J. Natl. Cancer. Inst. 85(7): 570574, 1993.

35. Kaur J. and Ralhan R., Differential expression of 70-kDa heat shock-protein in human oral tumorigenesis. Int. J. Cancer 63(6): 774-779, 1995.

36. Santarosa M., Favaro D., Quaia M. and Galligioni E., Expression of heat shock protein 72 in renal cell carcinoma: possible role and prognostic implications in cancer patients. Eur. J. Cancer 33(6): 873-877, 1997.

37. Motzer R.J., Bander N.H. and Nanus D.M., Renal-cell carcinoma. N. Engl. J. Med. 335(12): 865-875, 1996.

38. Yamazaki D., Kurisu S. and Takenawa T., Regulation of cancer cell motility through actin reorganization. Cancer Sci. 96(7): 379-386, 2005.

39. Fujita H., Okada F., Hamada J., Hosokawa M., Moriuchi T., Koya R.C. and Kuzumaki N., Gelsolin functions as a metastasis suppressor in B16-BL6 mouse melanoma cells and requirement of the carboxyl-terminus for its effect. Int. J. Cancer 93(6): 773-780, 2001. 